\title{
Comparisons of Breast Milk Fatty Acid Profiles in Overweight and Obese Women
}

\author{
Karim Karbin ${ }^{1}$, Mohammad Hashemi ${ }^{2,1}$, Gholamreza Khademi ${ }^{3}$, Lida Jarahi ${ }^{4}$, Golnaz Ranjbar ${ }^{1}$, Maryam Khosravi ${ }^{1}$, Mohsen Nematy ${ }^{5,1 *}$ \\ 1- Department of Nutrition, Faculty of Medicine, Mashhad University of Medical Sciences, Mashhad, Iran \\ 2- Medical Toxicology Research Center, Mashhad University of Medical Sciences, Mashhad, Iran \\ 3- Neonatal Research Center, Faculty of Medicine, Mashhad University of Medical Sciences, Mashhad, Iran \\ 4- Community Medicine Department, Faculty of Medicine, Mashhad University of Medical Sciences, Mashhad, Iran \\ 5- Metabolic Syndrome Research Center, Mashhad University of Medical Sciences, Mashhad, Iran
}

Received: July 2020

Accepted: October 2020

\section{A B S T R A C T}

Background and Objectives: Breast milk composition is affected by several factors such as maternal diet and body mass index. Milk fats include a major calorie content of the milk, which changes dramatically throughout the lactation. The aim of the present study was to assess breast milk fatty acid profile in overweight and obese mothers and its correlations with maternal body mass index.

Materials and Methods: Milk samples were collected from 73 healthy lactating women referring to various health centers in Mashhad. Data were collected using food frequency questionnaire to assess the maternal dietary intakes. Breast milk fatty acid profile was assessed using gas chromatography.

Results: Breast milk content of saturated fatty acids was higher in obese mothers than overweight mothers $(p=0.03)$ with positive correlations with maternal body mass index $(\mathrm{r}=0.256 ; p=0.037)$. Furthermore, breast milk monounsaturated fatty acids were negatively correlated with pre-pregnancy body mass index $(\mathrm{r}=-0.385 ; p=0.003)$ and pre-pregnancy body weight $(r=-0.276 ; p=0.026)$. However, no significant correlations were observed between the maternal body fat mass and fat proportion with the breast milk fatty acids $(p>0.05)$. Moreover, no significant associations were seen between dietary saturated fatty acids, milk monounsaturated fatty acids, polyunsaturated fatty acids, n-3 polyunsaturated fatty acids and n-6 polyunsaturated fatty acids with the corresponding fatty acids in breast milk $(p>0.05)$.

Conclusions: Based on the results, breast milk of the obese mothers included higher proportion of saturated fatty acids, while no significant differences were observed in the contents of milk monounsaturated fatty acids and polyunsaturated fatty acids between the overweight and obese women. Furthermore, maternal pre-pregnancy body mass index was negatively correlated with the contents of milk monounsaturated fatty acids, while it was positively correlated with saturated fatty acids contents.

Keywords: Human milk, Fatty acids, Body mass index, Obesity, Gas chromatography

\section{Introduction}

Breast milk is an optimal food for infants during the first six months of their lives (1). With its unique composition of essential nutrients and various bioactive factors, breast milk provides ideal nourishments to the infants. Nevertheless, the human breast milk composition may vary largely, particularly in its fat contents, which play key roles in development of the central nervous system (CNS) in infants, providing approximately $50 \%$ of the milk energy $(2,3)$. Previous studies have indicated that human milk composition is affected by the environmental factors, such as infant maturity (4), maternal nutritional status (5) and breastfeeding stage (6). Furthermore, maternal body mass index (BMI) has been reported to positively correlate with breast milk energy (7) and lipid contents $(8,9)$. Maternal diets can affect breast milk lipid components with no effects on total fats. Diets with high levels of polyunsaturated fatty acids (PUFAs) can increase the levels of PUFA in breast milks with no changes in total fat contents (10).

The suggested fatty acid (FA) ratio of human breast milks is nearly $30 \%$ of the maternal FA intakes, $70 \%$ of the FA reservoirs and the minimal quantities derived from de novo synthesis $(10,11)$. Panagos et al. reported that breast milk of mothers with overweight and obesity included 
lower n-3 PUFA (12). Similarly, other studies have reported that maternal obesity is associated with higher $n-6$ PUFA/n-3 PUFA ratios of the breast milks (12-14). General changes in dietary habits with increased intakes of n-6 PUFA compared to n-3 PUFA are reflected in breast milks (15). It is suggested that increased n-6 PUFA intakes might contribute to obesity epidemics (16). Moreover, studies have demonstrated that neonates supplemented with n-3 FAs have lower fat mass than those receiving no supplementations (17). Being overweight or obese are factors for having extra body fats. Although evidence suggest that breast milks of mothers with normal weights vary from those of overweight and obese for saturated fatty acids (SFA) (18), n-3 PUFA $(12,18,19)$ and n-6 PUFA/n3 PUFA ratios $(13,14,18)$, a few studies have addressed these variations in breast milk FA profiles in obese and overweight mothers. However, these studies did not explain distinct characteristics of the categories (20). Since FA reservoirs in the body are responsible for most breast milk FA variations (10), the aim of the present study was to investigate if FA compositions of the breast milks are similar in overweight and obese mothers.

\section{Materials and Methods}

\section{Participants}

This observational study was carried out on breastfeeding volunteers referred to health centers in Mashhad, Iran, September 2018 to March 2019. The inclusion criteria of the study were as follows: 1) ages of 18-55 years; 2) current BMI of $\geq 25 \mathrm{~kg} / \mathrm{m}^{2}$; 3) mothers with apparently healthy infants; and 4) neonatal ages of > 10 to $<90$ days. The exclusion criteria were as follows: 1) twin delivery/premature births (before 37 weeks of gestation); 2) history of gestational diabetes; 3) use of probiotics or n-3 supplements; and 4) alcohol/tobacco consumption. After receiving written informed consents from the participants, demographic data were collected, including mother age at delivery, mode of delivery, mode of breastfeeding, self-reported pre-pregnancy weight and height and weight gain during pregnancy as well as infant gender and birth weight. Obese women $\left(\mathrm{BMI} \geq 30 \mathrm{~kg} / \mathrm{m}^{2}\right.$ ) and a matched overweight group with BMI of 25-29.9 $\mathrm{kg} / \mathrm{m}^{2}$ were included in the study based on the World Health Organization (WHO) criteria. Matching was carried out based on maternal age and weeks postpartum between the overweight mothers from the same health centers. The study protocol was approved by the Ethics Committee of Mashhad University of Medical Sciences (code: IR.MUMS.MEDICAL.REC.1397.114).

\section{Sample size}

Sample size was calculated $(n=36)$ based on the study of Rudolph et al. ( $\left.Y^{\prime}\right)$ and the following formula. Where, $n$ was the predicted sample size, $\alpha$ was the probability of a type 1 error (0.05), $\beta$ was the probability of a type 2 error (0.1), S was S.D and $\bar{X}$ was the mean.

$$
n_{1}=n_{2}=\frac{\left(S_{1}{ }^{2}+S_{2}{ }^{2}\right)\left(Z_{1-\frac{\alpha}{2}}+Z_{1-\beta}\right)^{2}}{\left(\bar{X}_{1}-\bar{X}_{2}\right)^{2}}
$$

\section{Data collection}

Anthropometric measurements included maternal body weight, BMI and body fat proportion, using body composition analyzer (Tanita BC 418MA, Tokyo, Japan) at the inclusion. Height was measured using SECA Portable Stadiometer (SECA, Hamburg, Germany). The BMI classification by the World Health Organization (WHO) was used as follows: overweight, $25.0-29.9 \mathrm{~kg} / \mathrm{m}^{2}$; and obese, $>30 \mathrm{~kg} / \mathrm{m}^{2}$ (22). Selected participants were asked to collect their breast milk samples manually. First, they let a few drops of their breast milks to remove and then collected breast milks as much as possible (at least $20 \mathrm{ml}$ ), storing samples in plastic containers (18). Breast milk samples were collected from the fasting mothers (8:00 11:00 AM) by a trained expert and stored at $-20{ }^{\circ} \mathrm{C}$ until use. Maternal dietary intakes within the past 30 days were assessed using semi-quantitative food frequency questionnaire (FFQ) with 160 Iranian food items, validated by Nematy et al. (23). The completed FFQ were analyzed using United States Department of Agriculture (USDA) National Nutrient Database for Standard Reference Legacy Release (April 2018).

\section{Milk fatty acid analysis}

Breast milk FAs were extracted from the samples using modified Folch method $(24,25)$. In brief, $500 \mu \mathrm{l}$ of homogenized breast milk samples were added to a mixture of chloroform/methanol (v/v, 2:1) and $600 \mu \mathrm{l}$ of normal saline and vortexed for $1 \mathrm{~min}$. After centrifugation at 4,000 rpm for $4 \mathrm{~min}$, the lower phase was collected, and the solvent was evaporated using a rotary evaporator (Model Laborota 4003, Heidolph WB, Germany). Then, fatty acid methyl esters (FAME) were assessed through dissolving dried lipid extracts in n-heptane and methanolic $\mathrm{KOH}(2$ $\mathrm{N})$. The FAs were analyzed using gas chromatograph (Varian 450-GC, Varian Associates, Walnut Creek, CA, USA) equipped with a fused silica capillary column (length, $100 \mathrm{~m}$; film thickness, $0.25 \mathrm{~mm} \times 0.2 \mu \mathrm{m}$ ), which coated with silicon-based polymers (polysiloxane), polyethylene glycols and solid adsorbent (EU). Then, standard FAME mix (Supelco 37 Component FAME Mix, Sigma-Aldrich, USA) was injected prior to sample analysis to characterize FAs. Quantification was accomplished using peak areas, which were retrieved from the integrator coupled to the chromatograph. The FA data were reported as proportions of the total detected FAs. 


\section{Statistical analysis}

Data analysis was carried out using SPSS Software v.11.0 (SPSS, Chicago, IL, USA). Variable distributions were evaluated using Kolmogorov-Smirnov test. In addition, descriptive statistics (mean, standard deviation [SD], median and interquartile range) were used. Student's t-test and Mann-Whitney $U$ test were used to compare demographic data, anthropometric data and nutritional values of the maternal diets between the study groups. Furthermore, Pearson's or Spearman's correlationcoefficients were used to assess correlations between the breast milk compositions, maternal diets and body compositions based on data normality. Linear regression analysis was carried out to show if maternal BMI significantly predicted breast milk FA profiles. Maternal age, days postpartum at the time of sampling, mode of delivery, mode of breastfeeding, energy intake, and dietary FA intake were considered as confounders. In all the statistical analyses, $p$-values of less than 0.05 were considered as significant.

\section{Results}

In total, 73 eligible participants (37 overweight and 36 obese participants) enrolled in the study. Table 1 shows baseline demographic characteristics of the participants. No significant differences were seen between the groups in time points after birth when breast milk samples were collected (75.31 and 69.08 days for overweight and obese participants, respectively). In addition, no significant differences were observed between the study groups in mean maternal age, pregnancy weight gain and infant birth weight. No significant differences were reported in exclusive breastfeeding rate, mode of delivery and infant gender between the study groups. Table 2 shows data on breast milk FA compositions in overweight and obese women. The SFA (43\%) was the major constituent of breast milk FAs, whereas PUFA (23\%) included the lowest proportion. In addition, SFA contents of the breast milk samples were significantly higher in obese mothers, compared to overweight mothers $(p=0.03)$. Ratios of unsaturated to saturated fatty acids were lower in breast milk samples of the obese groups $(p=0.04)$. However, no significant differences were seen in ratios of other FAs, including MUFA, PUFA, n-6 PUFA, n-3 PUFA and n-6 PUFA/n-3 PUFA between the study groups $(p>0.05)$.

Based on the information in Table 3, total rates of the simple sugars in maternal diets of the participants were negatively correlated with the breast milk contents of PUFA ( $\mathrm{r}=-0.369, p=0.035)$, $\mathrm{n}-6$ PUFA $(\mathrm{r}=-0.370, p=$ $0.034)$ and PUFA/SFA ratio $(\mathrm{r}=-0.407, p=0.019)$. However, no significant correlations were reported between the dietary intakes of SFA, MUFA, PUFA, n-3 PUFA and n-6 PUFA in breast milk samples and their corresponding FAs $(p>0.05)$. In addition, energy, protein, carbohydrate, total lipids and dietary fiber intakes were not associated with the breast milk FA profiles $(p>0.05)$. Based on the information from Table 4 , maternal age was positively correlated with SFA $(r=0.250, p=0.042)$ and negatively with MUFA $(\mathrm{r}=-0.25, p=0.036)$, USFA/SFA ratio $(\mathrm{r}=-0.255, p=0.038)$ and MUFA/SFA ratio $(\mathrm{r}=-$ $0.304, p=0.012)$ in breast milk samples. It is noteworthy that associations between these parameters were significant after adjustment for maternal BMI. Moreover, maternal weight was negatively associated with MUFA/SFA ratios ( $\mathrm{r}=-0.251, p=0.040)$ in breast milk samples. Prepregnancy maternal weight was inversely correlated with MUFA $(\mathrm{r}=-0.276, p=0.026)$ and MUFA/SFA ratios $(\mathrm{r}=-$ $0.285, p=0.022)$.

Table 1. Demographic and anthropometric characteristics of the study groups

\begin{tabular}{|c|c|c|c|c|}
\hline \multirow{2}{*}{\multicolumn{2}{|c|}{ Characteristics }} & Overweight & Obese & \multirow{2}{*}{ p-value } \\
\hline & & $\mathrm{n}=37$ & $\mathrm{n}=36$ & \\
\hline \multicolumn{2}{|l|}{ Mother's age (years) } & $28.73 \pm 5.19$ & $30.83 \pm 4.65$ & 0.195 \\
\hline \multicolumn{2}{|c|}{ Prepregnancy weight (Kg) } & $66.24 \pm 7.87$ & $78.72 \pm 9.38$ & 0.001 \\
\hline \multicolumn{2}{|c|}{ Weight gain during pregnancy (Kg) } & $11.85 \pm 4.56$ & $13.3 \pm 5.31$ & 0.228 \\
\hline \multicolumn{2}{|c|}{ BMI $\left(\mathrm{kg} / \mathrm{m}^{2}\right)$} & $27.48 \pm 1.26$ & $33.51 \pm 2.74$ & 0.001 \\
\hline \multicolumn{2}{|l|}{ Body fat mass (Kg) } & $24.86 \pm 3.66$ & $35.31 \pm 5.09$ & $<0.001$ \\
\hline \multicolumn{2}{|c|}{ Body fat proportion (\%) } & $35.17 \pm 2.50$ & $42.04 \pm 2.87$ & $<0.001$ \\
\hline \multicolumn{2}{|c|}{ Infant age (day) } & $75.31 \pm 26.04$ & $69.08 \pm 26.35$ & 0.320 \\
\hline \multicolumn{2}{|l|}{ Birthweight (gr) } & $3344 \pm 334$ & $3278 \pm 564$ & 0.562 \\
\hline \multicolumn{2}{|c|}{ Exclusive breastfeeding (n)\% } & $26(70.2 \%)$ & $29(80.6 \%)$ & 0.580 \\
\hline \multirow[t]{2}{*}{ Infant gender $(\mathrm{n}) \%$} & Female & $20(54 \%)$ & $14(39 \%)$ & \multirow[b]{2}{*}{0.243} \\
\hline & Male & $17(46 \%)$ & $22(61 \%)$ & \\
\hline \multirow[t]{2}{*}{ Delivery type (n)\% } & Vaginal & $15(41 \%)$ & $16(44 \%)$ & \multirow{2}{*}{0.815} \\
\hline & Cesarean & $22(59 \%)$ & $20(56 \%)$ & \\
\hline
\end{tabular}

(Quantitative variables are expressed as mean \pm SD) 
Karim Karbin, et al: Breast milk fatty acid in overweight \& obese women

Table 2. Breast milk fatty acid compositions in overweight and obese women

\begin{tabular}{|c|c|c|c|c|c|c|c|}
\hline & Overweight & Obese & $\mathrm{p}$-value & & Overweight & Obese & p-value \\
\hline SFA (\%) & $41.75 \pm 5.42$ & $44.52 \pm 4.96$ & $0.03^{\ddagger}$ & C20:1 (\%) & $1.24(1.07,1.49)$ & $1.18(0.91,1.45)$ & $0.35^{\perp}$ \\
\hline C4:0 (\%) & $0.12(0.06,0.21)$ & $0.12(0.08,0.17)$ & $0.54^{\perp}$ & $\mathrm{C} 22: \ln 9(\%)$ & $0.16(0.06,0.44)$ & $0.08(0.05,0.32)$ & $0.43^{\perp}$ \\
\hline C8:0 (\%) & $0.05(0.03,0.08)$ & $0.04(0.02,0.08)$ & $0.15^{\perp}$ & $\mathrm{C} 24: 1(\%)$ & $0.10(0.07,0.11)$ & $0.09(0.08,0.10)$ & $0.71^{\perp}$ \\
\hline C10:0 (\%) & $0.90(0.67,1.18)$ & $0.90(0.75,1.28)$ & $0.57^{\perp}$ & PUFA (\%) & $23.23(19.44,24.11)$ & $21.15(17.43,25.50)$ & $0.17^{\perp}$ \\
\hline $\mathrm{C} 12: 0(\%)$ & $4.96 \pm 1.79$ & $5.35 \pm 2.08$ & $0.39^{*}$ & C18:2n6c (\%) & $21.90 \pm 4.33$ & $20.82 \pm 4.75$ & $0.31^{\ddagger}$ \\
\hline $\mathrm{C} 14: 0(\%)$ & $5.64 \pm 2.00$ & $6.50 \pm 2.12$ & $0.08^{\ddagger}$ & $\mathrm{C} 18: 3 \mathrm{n} 6(\%)$ & $0.19(0.15,0.22)$ & $0.22(0.19,0.24)$ & $0.18^{\perp}$ \\
\hline C15:0 (\%) & $0.26 \pm 0.09$ & $0.27 \pm 0.09$ & $0.43^{\ddagger}$ & C18:3n3 (\%) & $0.22(0.15,0.40)$ & $0.23(0.18,0.28)$ & $0.83^{\perp}$ \\
\hline $\mathrm{C} 16: 0(\%)$ & $23.24 \pm 3.31$ & $23.86 \pm 3.35$ & $0.43^{\ddagger}$ & $\mathrm{C} 20: 2(\%)$ & $0.32 \pm 0.11$ & $0.35 \pm 0.07$ & $0.14^{\ddagger}$ \\
\hline $\mathrm{C} 17: 0(\%)$ & $0.31(0.23,0.47)$ & $0.33(0.27,0.41)$ & $0.28^{\perp}$ & $\mathrm{C} 20: 3 \mathrm{n} 3(\%)$ & $0.43 \pm 0.13$ & $0.40 \pm 0.11$ & $0.34^{\ddagger}$ \\
\hline $\mathrm{C} 18: 0(\%)$ & $5.91 \pm 1.24$ & $6.09 \pm 0.81$ & $0.47^{\ddagger}$ & C20:4n6 (\%) & $0.04 \pm 0.02$ & $0.05 \pm 0.02$ & $0.40^{\ddagger}$ \\
\hline $\mathrm{C} 20: 0(\%)$ & $0.25 \pm 0.11$ & $0.22 \pm 0.10$ & $0.36^{\ddagger}$ & $\mathrm{C} 20: 5 \mathrm{n} 3(\%)$ & $0.03(0.02, .03)$ & $0.03(0.02,0.03)$ & $0.39^{\perp}$ \\
\hline C21:0 (\%) & $0.03 \pm 0.02$ & $0.02 \pm 0.01$ & $0.09^{\ddagger}$ & $\mathrm{C} 22: 2(\%)$ & $0.02(0.01,0.03)$ & $0.03(0.02,0.03)$ & $0.15^{\perp}$ \\
\hline C22:0 (\%) & $0.48(0.35,0.57)$ & $0.48(0.40,0.64)$ & $0.30^{\perp}$ & C22:6n3 (\%) & $0.04(0.02,0.07)$ & $0.03(0.02,0.05)$ & $0.11^{\perp}$ \\
\hline C23:0 (\%) & $0.05(0.03,0.14)$ & $0.06(0.04,0.10)$ & $0.67^{\perp}$ & USFA/SFA (ratio) & $1.35(1.19,1.65)$ & $1.22(1.06,1.48)$ & $0.04^{\perp}$ \\
\hline $\mathrm{C} 24: 0(\%)$ & $0.05 \pm 0.02$ & $0.06 \pm 0.02$ & $0.32^{\ddagger}$ & MUFA/SFA (ratio) & $0.82(0.74,1.01)$ & $0.74(0.66,0.86)$ & $0.05^{\perp}$ \\
\hline MUFA (\%) & $34.34(32.36,36.21)$ & $32.89(31.30,35.24)$ & $0.15^{\perp}$ & PUFA/SFA (ratio) & $0.55(0.43,0.62)$ & $0.47(0.39,0.63)$ & $0.08^{\perp}$ \\
\hline $\mathrm{C} 14: 1(\%)$ & $0.11 \pm 0.06$ & $0.12 \pm 0.07$ & $0.55^{\ddagger}$ & $\begin{array}{l}\text { PUFA/MUFA } \\
\text { (ratio) }\end{array}$ & $0.67 \pm 0.17$ & $0.66 \pm 0.20$ & 0.74 \\
\hline C16:1 (\%) & $1.22(0.98,1.86)$ & $1.28(1.01,1.52)$ & $0.84^{\perp}$ & n-3 PUFA (\%) & $0.68 \pm 0.29$ & $0.61 \pm 0.23$ & $0.23^{\ddagger}$ \\
\hline C17:1 (\%) & $0.26(0.20,0.33)$ & $0.26(0.21,0.39)$ & $0.64^{\perp}$ & n-6 PUFA (\%) & $22.33(18.63,23.61)$ & $20.63(16.99,24.88)$ & $0.20^{\perp}$ \\
\hline $\mathrm{C} 18: \ln 9 \mathrm{c}(\%)$ & $30.85(28.95,32.87)$ & $30.21(28.3,32.5)$ & $0.33^{\perp}$ & & & & \\
\hline
\end{tabular}

Values presented as proportions from all fatty acids (mean \pm SD or Median (IQR)). $p<0.05$ were considered statistically significant. Statistical tests: Student's t-test $\$$, Mann-Whitney U test $\perp$

Table 3. Correlations between the maternal dietary intakes and the breast milk fatty acids

\begin{tabular}{|c|c|c|c|c|c|c|c|c|c|c|c|}
\hline \multirow[b]{2}{*}{ Dietary intake } & \multicolumn{11}{|c|}{ Breast milk fatty acid composition } \\
\hline & & SFA $(\%)$ & MUFA (\%) & PUFA (\%) & $\begin{array}{l}\text { USFA/SFA } \\
\text { ratio }\end{array}$ & $\begin{array}{l}\text { MUFA/SF } \\
\text { A ratio }\end{array}$ & $\begin{array}{l}\text { PUFA/SFA } \\
\text { ratio }\end{array}$ & $\begin{array}{l}\text { PUFA/MU } \\
\text { FA ratio }\end{array}$ & $\begin{array}{l}\text { n-3 PUFA } \\
(\%)\end{array}$ & $\begin{array}{l}\text { n-6 PUFA } \\
(\%)\end{array}$ & $\begin{array}{c}\mathrm{n}-6 \\
\text { PUFA/n-3 } \\
\text { PUFA ratio }\end{array}$ \\
\hline Energy (Kcal) & $\mathrm{r}$ & 0.216 & 0.011 & -0.262 & -0.249 & -0.137 & -0.302 & -0.211 & -0.056 & -0.258 & -0.173 \\
\hline Protein $(E I \%)$ & $\mathrm{r}$ & -0.027 & -0.095 & 0.139 & 0.029 & -0.052 & 0.126 & 0.131 & -0.193 & 0.150 & 0.303 \\
\hline Total lipid (EI\%) & $\mathrm{r}$ & 0.086 & 0.303 & 0.279 & -0.069 & -0.230 & 0.165 & 0.307 & 0.046 & 0.276 & 0.156 \\
\hline Carbohydrate (EI\%) & $\mathrm{r}$ & -0.107 & 0.306 & -0.219 & 0.093 & 0.220 & -0.113 & -0.319 & 0.023 & -0.220 & -0.115 \\
\hline Fiber (g/1000Kcal) & $\mathrm{r}$ & -0.013 & 0.002 & 0.014 & -0.017 & -0.013 & -0.016 & 0.028 & 0.224 & 0.001 & -0.158 \\
\hline Starch (EI\%) & $\mathrm{r}$ & -0.162 & -0.063 & 0.262 & 0.145 & 0.033 & 0.240 & 0.249 & -0.169 & 0.272 & 0.289 \\
\hline Sugars, total $(\mathrm{EI} \%)$ & $\mathrm{r}$ & 0.080 & 0.263 & $-0.369^{*}$ & -0.087 & 0.097 & -0.287 & $-0.407 *$ & 0.049 & $-0.370 *$ & -0.023 \\
\hline Cholesterol (mg) & $\mathrm{r}$ & 0.230 & -0.066 & -0.195 & -0.259 & -0.187 & -0.254 & -0.136 & -0.081 & -0.190 & -0.056 \\
\hline Trans fatty acids (EI\%) & $\mathrm{r}$ & -0.015 & -0.054 & 0.076 & 0.039 & -0.003 & 0.078 & 0.083 & 0.122 & 0.068 & -0.181 \\
\hline SFA (EI\%) & $\mathrm{r}$ & 0.073 & -0.101 & 0.034 & -0.075 & -0.100 & -0.014 & 0.081 & -0.022 & 0.035 & 0.123 \\
\hline MUFA (EI\%) & $\mathrm{r}$ & -0.184 & -0.097 & 0.311 & 0.186 & 0.050 & 0.291 & 0.286 & 0.283 & 0.293 & -0.127 \\
\hline PUFA (EI\%) & $\mathrm{r}$ & -0.023 & -0.059 & 0.095 & 0.048 & 0.018 & 0.072 & 0.152 & -0.006 & 0.095 & -0.015 \\
\hline USFA/SFA ratio & $\mathrm{r}$ & -0.004 & 0.029 & -0.031 & 0.012 & 0.027 & -0.012 & -0.027 & -0.019 & -0.030 & -0.118 \\
\hline MUFA/SFA ratio & $\mathrm{r}$ & -0.016 & 0.012 & 0.001 & 0.032 & 0.031 & 0.022 & 0.001 & 0.023 & 0.000 & -0.180 \\
\hline PUFA/SFA ratio & $\mathrm{r}$ & -0.029 & 0.003 & 0.025 & 0.037 & 0.027 & 0.035 & 0.036 & 0.022 & 0.024 & -0.116 \\
\hline PUFA/MUFA ratio & $\mathrm{r}$ & -0.119 & 0.025 & 0.112 & 0.112 & 0.081 & 0.111 & 0.120 & 0.110 & 0.105 & -0.061 \\
\hline n-3 PUFA (EI\%) & $\mathrm{r}$ & -0.059 & -0.098 & 0.183 & 0.087 & 0.011 & 0.155 & 0.208 & 0.171 & 0.172 & -0.215 \\
\hline n-6 PUFA (EI\%) & $\mathrm{r}$ & -0.015 & -0.077 & 0.105 & 0.033 & -0.012 & 0.082 & 0.148 & -0.031 & 0.107 & 0.018 \\
\hline $\begin{array}{l}\text { n-6 PUFA/n-3 PUFA } \\
\text { ratio }\end{array}$ & $\mathrm{r}$ & 0.050 & -0.105 & 0.046 & -0.070 & -0.102 & -0.001 & 0.091 & -0.135 & 0.055 & 0.187 \\
\hline
\end{tabular}

The results are shown with Pearson's correlation coefficients. The statistically significant correlations are shown as highlights $(* \mathrm{p}<0.05, * * \mathrm{p}<0.01)$.

Table 4. Correlations between the maternal anthropometric characteristics and the breast milk fatty acids

\begin{tabular}{|c|c|c|c|c|c|c|c|c|c|}
\hline $\begin{array}{l}\text { Breast milk fatty acic } \\
\text { composition }\end{array}$ & & $\begin{array}{l}\text { Mother's age } \\
\text { (years) }\end{array}$ & $\begin{array}{c}\text { Prepregnancy } \\
\text { weight }(\mathrm{Kg})\end{array}$ & $\begin{array}{c}\text { Weight gain } \\
\text { during pregnancy } \\
(\mathrm{Kg})\end{array}$ & $\begin{array}{c}\text { Maternal } \\
\text { weight }(\mathrm{Kg})\end{array}$ & $\begin{array}{l}\text { Prepregnancy } \\
\text { BMI }(\mathrm{kg} / \mathrm{m} 2)\end{array}$ & BMI $\left(\mathrm{kg} / \mathrm{m}^{2}\right)$ & $\begin{array}{l}\text { Body fat mass } \\
\qquad(\mathrm{Kg})\end{array}$ & $\begin{array}{c}\text { Body fat } \\
\text { proportion } \\
(\%)\end{array}$ \\
\hline SFA & $r$ & $0.250^{*}$ & 0.211 & 0.137 & 0.212 & $\begin{array}{l}0.285^{*} \\
\end{array}$ & $0.256^{*}$ & 0.226 & 0.214 \\
\hline MUFA & $\mathrm{r}$ & $-0.256^{*}$ & $-0.276^{*}$ & 0.132 & -0.175 & $-0.358 * *$ & -0.224 & -0.151 & -0.096 \\
\hline PUFA & $\mathrm{r}$ & -0.142 & -0.081 & -0.189 & -0.116 & -0.125 & -0.167 & -0.165 & -0.209 \\
\hline USFA/SFA & $\mathrm{r}$ & $-0.255^{*}$ & -0.211 & -0.135 & -0.212 & $-0.287 *$ & $-0.256^{*}$ & -0.226 & -0.212 \\
\hline MUFA/SFA & $\mathrm{r}$ & $-0.304^{*}$ & $-0.285^{*}$ & -0.066 & $-.251^{*}$ & $-0.351 * *$ & $-0.277 *$ & -0.235 & -0.183 \\
\hline PUFA/SFA & $\mathrm{r}$ & -0.177 & -0.139 & -0.180 & -0.169 & -0.205 & -0.221 & -0.208 & -0.232 \\
\hline PUFA/MUFA & $\mathrm{r}$ & -0.013 & 0.009 & -0.231 & -0.069 & 0.029 & -0.077 & -0.114 & -0.169 \\
\hline $\mathrm{n}-3$ PUFA & $\mathrm{r}$ & 0.106 & -0.053 & 0.033 & -0.080 & 0.037 & -0.044 & -0.011 & 0.077 \\
\hline n-6 PUFA & $\mathrm{r}$ & -0.152 & -0.072 & -0.189 & -0.103 & -0.117 & -0.157 & -0.155 & -0.205 \\
\hline n-6 PUFA/n-3 PUFA & $\mathrm{r}$ & -0.174 & -0.052 & -0.162 & 0.032 & -0.116 & -0.009 & -0.025 & -0.101 \\
\hline
\end{tabular}

The results are shown with Spearman correlation coefficients. The statistically significant correlations are shown as highlights $(* \mathrm{p}<0.05, * * \mathrm{p}<0.01)$. 
In this study, maternal pre-pregnancy BMI was positively correlated with SFA $(r=0.285, p=0.019)$ and negatively with MUFA $(\mathrm{r}=-0.385, p=0.003)$, USFA/SFA ratios $(\mathrm{r}=-0.287, p=0.019)$ and MUFA/SFA ratios $(\mathrm{r}=-$ $0.351, p=0.004)$ in breast milks. During sample collection, the current maternal BMI was positively correlated with SFA $(r=0.256, p=0.037)$ and negatively with USFA/SFA $(\mathrm{r}=-0.266, p=0.036)$ and MUFA/SFA $(\mathrm{r}=-0.277, p=$ $0.023)$ ratios. However, no significant associations were seen between the body fat mass, fat proportion, weight gain during pregnancy and breast milk FA profile $(p>0.05)$. Results of linear regression (Table 5) revealed that maternal BMI significantly contributed to the model that predicted SFA $(\beta=0.392, p=0.023)$ and USFA/SFA ratios $(\beta=-0.022, p=0.027)$ in breast milks. The current maternal BMI was not a significant predictor of breast milk FA profiles after adjustment of the confounding factors (maternal age, days postpartum at the time of sampling, mode of delivery, mode of breastfeeding, energy intake and dietary intake of each FA).

\section{Discussion}

Based on the present results, SFA was the major fraction of breast milks (43\%) and included significant differences between obese and overweight mothers as well as the USFA/SFA ratios. Although PUFA included the smallest proportion of breast milk FAs, the averages of total PUFA and linoleic acid proportions were higher (22.3 and 21.3\%, respectively) than those reported in the previous studies $(19,26)$. However, no significant differences were seen in breast milk FAs between the groups. Adiposity measurements and higher BMI have been associated with the higher total fat contents of breast milks (27). However, a few studies have compared the breast milk FA profiles between the obese and overweight mothers, suggesting inconsistent results. Although the breast milk ratios of n-6 PUFA/n-3 PUFA have been higher in obese and overweight mothers than normal-weight mothers (12-14, 18), no significant differences were observed in $n-6$ PUFA $/ n-3$ PUFA ratios of breast milks between the overweight and obese mothers. The current maternal BMI was positively correlated with SFA and negatively with USFA/SFA ratios. Maternal BMI contributed to the linear regression model that predicted SFA and USFA/SFA ratios. However, the BMI effects were not significant after adjusting for confounding factors. In a similar study, Makela et al. reported that breast milk SFA contents were significantly higher $(p=0.012)$ and n-3 PUFA FAs $(p=0.010)$ were significantly lower in overweight mothers than normal-weight mothers. However, no significant associations were reported between MUFA and n-6 PUFA (18).

In the present study, maternal age and pre-pregnancy BMI were positively correlated with SFA and negatively with MUFA contents of the human breast milks. However, no significant correlations were seen between the PUFA, n3 PUFA, n-6 PUFA contents as well as the n-6 PUFA/n-3 PUFA ratios in breast milk samples with maternal anthropometric indices. Garcia-Ravelo et al. reported low concentrations of n-3 long-chain polyunsaturated fatty acids (LCPUFA), including eicosapentaenoic acid (EPA) $(p=0.005)$ and docosahexaenoic acid $(p=0.015)$, in breast milks of overweight women (28). In contrast, breast milk contents of SFA, MUFA, total n-6 PUFA, total n-3 PUFA, and free FAs included no significant differences between the normal and overweight mothers (13). In contrast to maternal weight and BMI, no significant associations were reported between the maternal body fat masses, fat proportions and breast milk FA profiles. Therefore, it could be concluded that maternal body fat masses and visceral fats increased regardless of postpartum weight losses (29). Human milk FAs are either synthesized by mammary alveolar epithelia or originate from the blood plasma (10). Availability of the circulating FAs in mammary glands, which is affected by the maternal fat reservoirs and dietary habits, determines FA profiles of the breast milks (30-32). In a study by Ahmadi et al., obesity was negatively associated with plasma n-3 PUFA (33). However, the SFA content of breast milks increased as lipids were synthesized from the tissues when breastfeeding mothers consumed excessive non-fat calories (10).

Table 5. Linear regression models for assessing associations between the maternal BMI and the breast milk fatty acids

\begin{tabular}{|c|c|c|c|c|}
\hline Variables & $\begin{array}{c}\text { Unadjusted model } \\
\beta[95 \% \mathrm{CI}]\end{array}$ & p-Value & $\begin{array}{c}\text { Adjusted model } \\
\beta[95 \% \mathrm{CI}]\end{array}$ & p-Value \\
\hline SFA & $0.392\left[0.056 \_0.728\right]$ & $0.023^{*}$ & $0.449\left[-0.206 \_1.104\right]$ & 0.170 \\
\hline MUFA & $-0.146[-0.399-0.108]$ & 0.255 & $-0.043[-0.651-0.565]$ & 0.885 \\
\hline PUFA & $-0.156\left[-0.454 \_0.152\right]$ & 0.316 & $-0.383\left[-1.053 \_0.288\right]$ & 0.250 \\
\hline USFA/SFA & $-0.022\left[-0.041_{-}-0.003\right]$ & $0.027 *$ & $-0.022\left[-0.063 \_0.018\right]$ & 0.260 \\
\hline MUFA/SFA & $-0.012\left[-0.024 \_0\right]$ & 0.054 & $-0.001\left[-0.032 \_0.031\right]$ & 0.965 \\
\hline PUFA/SFA & $-0.008\left[-0.019 \_0.003\right]$ & 0.158 & $-0.014\left[-0.040 \_0.012\right]$ & 0.276 \\
\hline PUFA/MUFA & $-0.001\left[-0.013 \_0.011\right]$ & 0.868 & $-0.018\left[-0.048 \_0.011\right]$ & 0.213 \\
\hline n-3 PUFA & $0\left[-0 .-13 \_0.018\right]$ & 0.961 & $0.003\left[-0.038 \_0.043\right]$ & 0.900 \\
\hline n-6 PUFA & $-0.155\left[-0.464 \_0.153\right]$ & 0.318 & $-0.365\left[-1.041 \_0.311\right]$ & 0.276 \\
\hline n-6 PUFA/n-3 PUFA & $0.229\left[-0.606 \_1.065\right]$ & 0.585 & $0.596\left[-1.584 \_2.776\right]$ & 0.573 \\
\hline
\end{tabular}

The statistically significant correlations are shown as highlights $(* \mathrm{p}<0.05)$. 
Although SFA is suggested to increase low-density lipoprotein cholesterol (LDL-C) and increase cardiovascular disease (CVD) risks (34), evidence have shown no associations between higher intakes of total fats or SFAs with growth, adiposity and cardiometabolic health in children up to 6 years of age (35). However, higher plasma levels of long-chain saturated fatty acids (LCSFA) and high n-3 and low n-6 LCPUFA patterns were positively associated with psychomotor development scores in infants up to six months of age (36). Laiho et al. suggested that the proportion of total SFA might be an important regulator of breast milk immune milieu as it was negatively associated with TGF-b2 levels (37). Naturally, maternal diets affect constituents of the breast milk lipids (10). Although breast milk FAs are predominantly composed of the FAs deposited in fat tissues, maternal diets might affect breast milk FA contents, especially its PUFA contents. Diets with fish and seafood increase the intake of n-3 PUFA FAs, EPA and DHA as well as concentration of the corresponding FAs expressed in breast milks $(20,38)$. Based on the results of the present study, dietary intakes included no significant differences between the overweight and obese mothers, except for the total energy intakes $(p=0.014)$. In addition, no significant correlations were observed between the breast milk FAs and energy intakes, carbohydrates, proteins, SFA, MUFA, PUFA, n-3 PUFA and n-6 PUFA in maternal diets. However, the breast milk levels of PUFA and n-6 PUFA were negatively correlated with the total sugar intakes of the mothers. These differences could be attributed to various breast milk FA extraction methods and maternal diet analyses. Therefore, genetic differences in FA desaturases should be considered important, affecting breast milk fat contents $(39,40)$. Studies have reported that maternal age is positively correlated with breast milk fat contents, especially colostrum and transitional milk (41). Antonakou et al. reported that MUFA levels in breast milks were negatively correlated with maternal ages (20). In the present study, increased maternal age was similarly associated with decreased MUFA and increased SFA breast milk contents. Although the exact mechanisms are still unclear, dietary habits and gut microbiome differences may be involved in such changes (20). One of the limitations of the present study included lack of local food composition databases for the maternal diet nutritional assessments.

\section{Conclusion}

The current findings supported the hypothesis that maternal weight and BMI could affect breast milk FA compositions. Although no significant differences were observed in MUFA and PUFA contents of the breast milks, breast milks of the obese mothers included higher proportions of SFA and lower ratios of unsaturated to saturated fatty acids, compared to that the breast milks of the overweight mothers did. This could be linked to differences in the metabolic functions between the obese and overweight mothers. Considering similar dietary habits in the two study groups, maternal diets were not significant affecting factors in FA contents of the breast milks.

\section{Acknowledgement}

The authors express their gratitude to Mashhad University of Medical Sciences for the financial supports of this study (grant no: 970082). Moreover, the authors would like to thank Mr. Farkhani for assisting this study.

\section{Financial disclosure}

The authors declared no financial interest.

\section{References}

1. WHO. Up to what age can a baby stay well nourished by just being breastfed? 2015 [updated July 2015 Available from: https://www.who.int/features/qa/21/en/.

2. Jenness R. Composition of milk. Fundamentals of dairy chemistry: Springer; 1988. p. 1-38.

3. Jensen RG. Lipids in human milk. Lipids. 1999;34(12):124371.

4. Charpak N, Ruiz J, team MMSobotK. Breast milk composition in a cohort of pre-term infants' mothers followed in an ambulatory programme in Colombia. Acta Paediatrica. 2007;96(12):1755-9.

5. Lönnerdal B. Effects of maternal dietary intake on human milk composition. The Journal of nutrition. 1986;116(4):499513.

6. Kulski J, Hartmann P. Changes in human milk composition during the initiation of lactation. Australian Journal of Experimental Biology and Medical Science. 1981;59(1):10114.

7. Bzikowska-Jura A, Czerwonogrodzka-Senczyna A, Oledzka G, Szostak-Wegierek D, Weker H, Wesolowska A. Maternal Nutrition and Body Composition During Breastfeeding: Association with Human Milk Composition. Nutrients. 2018;10(10).

8. Kim H, Kang S, Jung BM, Yi H, Jung JA, Chang N. Breast milk fatty acid composition and fatty acid intake of lactating mothers in South Korea. Br J Nutr. 2017;117(4):556-61.

9. Fujimori M, Franca EL, Fiorin V, Morais TC, Honorio-Franca AC, de Abreu LC. Changes in the biochemical and immunological components of serum and colostrum of overweight and obese mothers. BMC pregnancy and childbirth. 2015;15:166.

10. Lawrence RA, Lawrence RM. Breastfeeding : a guide for the medical profession. 2016:91-145.

11. Ailhaud G, Massiera F, Weill P, Legrand P, Alessandri JM, Guesnet P. Temporal changes in dietary fats: role of $n-6$ polyunsaturated fatty acids in excessive adipose tissue development and relationship to obesity. Progress in lipid research. 2006;45(3):203-36. 
12. Panagos PG, Vishwanathan R, Penfield-Cyr A, Matthan NR, Shivappa N, Wirth MD, et al. Breastmilk from obese mothers has pro-inflammatory properties and decreased neuroprotective factors. Journal of perinatology : official journal of the California Perinatal Association. 2016;36(4):284-90.

13. Garcia-Ravelo S, Diaz-Gomez NM, Martin MV, DortaGuerra R, Murray M, Escuder D, et al. Fatty Acid Composition and Eicosanoid Levels (LTE4 and PGE2) of Human Milk from Normal Weight and Overweight Mothers. Breastfeed Med. 2018;13(10):702-10.

14. Storck Lindholm E, Strandvik B, Altman D, Moller A, Palme Kilander C. Different fatty acid pattern in breast milk of obese compared to normal-weight mothers. Prostaglandins, leukotrienes, and essential fatty acids. 2013;88(3):211-7.

15. Ailhaud G, Guesnet P. Fatty acid composition of fats is an early determinant of childhood obesity: a short review and an opinion. Obesity reviews. 2004;5(1):21-6.

16. Sanders TA. Polyunsaturated fatty acids in the food chain in Europe. The American journal of clinical nutrition. 2000;71(1 Suppl):176S-8S.

17. Muhlhausler B, Gibson R, Makrides M. The effect of maternal omega-3 long-chain polyunsaturated fatty acid (n-3 LCPUFA) supplementation during pregnancy and/or lactation on body fat mass in the offspring: a systematic review of animal studies. Prostaglandins, Leukotrienes and Essential Fatty Acids. 2011;85(2):83-8

18. Makela J, Linderborg K, Niinikoski H, Yang B, Lagstrom H. Breast milk fatty acid composition differs between overweight and normal weight women: the STEPS Study. European journal of nutrition. 2013;52(2):727-35.

19. Sinanoglou VJ, Cavouras D, Boutsikou T, Briana DD, Lantzouraki DZ, Paliatsiou S, et al. Factors affecting human colostrum fatty acid profile: A case study. PLoS One. 2017;12(4):e0175817.

20. Antonakou A, Skenderi KP, Chiou A, Anastasiou CA, Bakoula C, Matalas AL. Breast milk fat concentration and fatty acid pattern during the first six months in exclusively breastfeeding Greek women. European journal of nutrition. 2013;52(3):963-73.

21. Rudolph MC, Young BE, Lemas DJ, Palmer CE, Hernandez TL, Barbour LA, et al. Early infant adipose deposition is positively associated with the n- 6 to $n-3$ fatty acid ratio in human milk independent of maternal BMI. International journal of obesity (2005). 2017;41(4):510-7.

22. Status WP. The use and interpretation of anthropometry. WHO technical report series. 1995;854(9).

23. Nematy M, Nouri M, Ghazizahedi S, Norouzy A, Mohajeri S, Shalaei N, et al. Validity and reproducibility of Iranian food frequency questionnaire. Switz Res Park J. 2013;102:2137-46.

24. Folch J, Lees M, Sloane Stanley GH. A simple method for the isolation and purification of total lipides from animal tissues. J Biol Chem. 1957;226(1):497-509.

25. Visentin S, Vicentin D, Magrini G, Santandreu F, Disalvo L, Sala $M$, et al. Red blood cell membrane fatty acid composition in infants fed formulas with different lipid profiles. Early Hum Dev. 2016;100:11-5.

26. Bahrami G, Rahimi Z. Fatty acid composition of human milk in Western Iran. Eur J Clin Nutr. 2005;59(4):494-7.

27. Leghi GE, Netting MJ, Middleton PF, Wlodek ME, Geddes DT, Muhlhausler BS. The impact of maternal obesity on human milk macronutrient composition: A systematic review and meta-analysis. Nutrients. 2020;12(4):934.

28. Chan D, Goruk S, Becker AB, Subbarao P, Mandhane PJ, Turvey SE, et al. Adiponectin, leptin and insulin in breast milk: associations with maternal characteristics and infant body composition in the first year of life. International journal of obesity (2005). 2018;42(1):36-43.

29. Cho GJ, Yoon HJ, Kim EJ, Oh MJ, Seo HS, Kim HJ. Postpartum changes in body composition. Obesity (Silver Spring). 2011;19(12):2425-8.

30. Armand M, Bernard JY, Forhan A, Heude B, Charles MA, group Em-ccs. Maternal nutritional determinants of colostrum fatty acids in the EDEN mother-child cohort. Clin Nutr. 2018;37(6 Pt A):2127-36.

31. Craig-Schmidt MC, Weete JD, Faircloth SA, Wickwire MA, Livant EJ. The effect of hydrogenated fat in the diet of nursing mothers on lipid composition and prostaglandin content of human milk. The American journal of clinical nutrition. 1984;39(5):778-86.

32. Jayakumar A, Tai MH, Huang WY, al-Feel W, Hsu M, AbuElheiga L, et al. Human fatty acid synthase: properties and molecular cloning. Proceedings of the National Academy of Sciences of the United States of America. 1995;92(19):86959.

33. Ahmadi F, Nematy M, Shafiee M, Arabi SM, Norouzy A, Tayefi M, et al. Overall and Abdominal Obesity Are Inversely Associated With Plasma n-3 Polyunsaturated Fatty Acids in Iranian Adults. Journal of Nutrition, Fasting and Health. 2018;6(4):198-204.

34. Givens DI. Saturated fats, dairy foods and health: A curious paradox? Nutrition Bulletin. 2017;42(3):274-82.

35. Stroobant W, Braun KV, Kiefte-de Jong JC, Moll HA, Jaddoe VW, Brouwer IA, et al. Intake of Different Types of Fatty Acids in Infancy Is Not Associated with Growth, Adiposity, or Cardiometabolic Health up to 6 Years of Age. The Journal of nutrition. 2017;147(3):413-20.

36. Siziba LP, Baumgartner J, Ricci C, Jacobs A, Rothman M, Matsungo TM, et al. Associations of plasma total phospholipid fatty acid patterns with feeding practices, growth, and psychomotor development in 6-month-old South African infants. Maternal \& child nutrition. 2019;15(3):e12763.

37. Laiho K, Lampi A-M, Hämäläinen M, Moilanen E, Piironen $\mathrm{V}$, Arvola T, et al. Breast milk fatty acids, eicosanoids, and cytokines in mothers with and without allergic disease. Pediatric research. 2003;53(4):642-7.

38. Lane KE, Derbyshire E. Systematic review of omega-3 enriched foods and health. British Food Journal. 2014;116(1):165-79. 
39. Guillou H, Zadravec D, Martin PG, Jacobsson A. The key roles of elongases and desaturases in mammalian fatty acid metabolism: Insights from transgenic mice. Progress in lipid research. 2010;49(2):186-99.

40. Smith S, Gagne HT, Pitelka DR, Abraham S. The effect of dietary fat on lipogenesis in mammary gland and liver from lactating and virgin mice. The Biochemical journal. 1969;115(4):807-15.
41. Dritsakou K, Liosis G, Valsami G, Polychronopoulos E, Skouroliakou M. The impact of maternal- and neonatalassociated factors on human milk's macronutrients and energy. The journal of maternal-fetal \& neonatal medicine : the official journal of the European Association of Perinatal Medicine, the Federation of Asia and Oceania Perinatal Societies, the International Society of Perinatal Obstet. 2017;30(11):1302-8. 